\title{
GMR
}

\section{Molecular characterization of carbapenemase genes in Acinetobacter baumannii in China}

\author{
F. Fang ${ }^{1 *}$, S. Wang ${ }^{2 *}$, Y.X. Dang ${ }^{3}$, X. Wang ${ }^{3}$ and G.Q. Yu ${ }^{3}$ \\ ${ }^{1}$ The CT Room, Nanyang City Center Hospital, Nanyang, China \\ 2Department of Endocrinology, Nanyang City Center Hospital, Nanyang, China \\ ${ }^{3}$ Department of Nursing, Nanyang City Center Hospital, Nanyang, China \\ *These authors contributed equally to this study. \\ Corresponding author: G.Q. Yu \\ E-mail: gqyu66@163.com \\ Genet. Mol. Res. 15 (1): gmr.15017432 \\ Received August 12, 2015 \\ Accepted December 3, 2015 \\ Published March 31, 2016 \\ DOI http://dx.doi.org/10.4238/gmr.15017432
}

ABSTRACT. Acinetobacter baumannii is an aerobic non-motile Gramnegative coccobacillus, and it is one of the most important nosocomial pathogens worldwide. The aim of this study was to determine the molecular epidemiology of the outbreak strains. Between March 2011 and March 2014, a total of 205 strains of $A$. baumannii were isolated from patients at the Nanyang City Center Hospital. The blaOXA-23, blaOXA-24, blaOXA-51, and blaOXA-58 genes were amplified by multiplex polymerase chain reaction. We found that $68(33.17 \%)$ strains were positive for the blaOXA-23 gene, and $88.24 \%$ of these 68 showed resistance to carbapenems, while $11.76 \%$ were sensitive to carbapenems. The blaOXA-51 gene was found in $132(64.39 \%)$ strains, and $17.42 \%$ of these were resistant to carbapenems while $82.58 \%$ were sensitive to carbapenems. Moreover, $5(2.44 \%)$ strains were positive for blaOXA-58, of which $80 \%$ were resistant to carbapenems and $20 \%$ were sensitive to carbapenems. We found that $A$. baumanni showed $100 \%$ drug resistance to ampicillin, cefotetan, cefazolin, and cefoperazone. Our findings suggest that the blaOXA-23 and blaOXA-51 
genes are most frequently identified in A. baumannii, while blaOXA-23 is the most important gene for resistance to carbapenems.

Key words: blaOXA-23; blaOXA-51; Acinetobacter baumannii; Carbapenems

\section{INTRODUCTION}

Acinetobacter baumannii is an aerobic non-motile, Gram-negative coccobacillus that is regarded as one of the most important nosocomial pathogens worldwide (Boucher et al., 2009). A. baumannii is associated with many nosocomial infections, such as pneumonia, bloodstream infections, septicemia, and urinary tract and wound infections (Gaynes et al., 2005; Dijkshoorn et al., 2007). Multidrug-resistance in A. baumannii is on the rise in hospitals worldwide (Dijkshoorn et al., 2007; Peleg et al., 2008). Carbapenems are often used to treat multidrug-resistant $A$. baumannii infections; however, an increasing number of studies have reported the outbreak of carbapenemresistant $A$. baumannii, thus creating a serious global therapeutic problem (Jeong et al., 2006; Yang et al., 2009; Peymani et al., 2012).

Carbapenem resistance arises through the recruitment and production of carbapenemhydrolyzing class $D \beta$-lactamase (CHDLs) or metallo- $\beta$-lactamase. In $A$. baumannii, 4 subgroups of acquired CHDLs have been found, including blaOXA-23, blaOXA-24, blaOXA-51, and blaOXA-58 (Poirel and Nordmann, 2006). Although these enzymes are weak hydrolyzers of carbapenems, drug resistance can develop through overexpression of the blaOXA genes. The aim of this study is to determine the molecular epidemiology of the outbreak strains.

\section{MATERIAL AND METHODS}

Between March 2011 and March 2014, a total of 205 strains of $A$. baumannii were isolated from infected patients at the Nanyang City Center Hospital. The strains were collected from patients who did not receive antibiotic therapy. All the samples were collected and stored at $-80^{\circ} \mathrm{C}$.

\section{DNA extraction and gene analysis}

The DNA of $A$. baumannii was extracted using the TIANamp Bacteria DNA Kit (Tiangen Biotech Co., Ltd., Beijing, China). Polymerase chain reaction (PCR) amplification was performed using Taq PCR Master Mix (Shanghai Lifefeng Biotech Co., Ltd., Beijing, China). The blaOXA-23, blaOXA-24, blaOXA-51, and blaOXA-58 genes were amplified by multiplex PCR. The primer sequences are listed in Table 1.

Table 1. PCR primers for PCR.

\begin{tabular}{l|l|l|r}
\hline OXA gene & Primer & Sequence & Amplicon size (bp) \\
\hline blaOXA-23 & OXA-23-like F & GATCGGATTGGAGAACCAGA & 501 \\
\hline blaOXA-24 & OXA-23-like R & ATTTCTGACCGCATTTCCAT & 1024 \\
\hline blaOXA-51 & OXA-24-like F & TTCCCCTAACATGAATTTG & 353 \\
\hline & OXA-24-like R & GTACTAATCAAAGTTGTGAA & \\
\hline blaOXA-58 & OXA-51-like F & TAATGCTTTGATCGGCCTTG & 507 \\
\hline & OXA-51-like R & TGGATTGCACTTCATCTTGG & \\
\hline
\end{tabular}


The PCRs were carried out in a total reaction volume of $50 \mu \mathrm{L}$, consisting of $25 \mu \mathrm{L}$ Taq Mix, $1 \mu \mathrm{L}$ primers, $1 \mu \mathrm{L}$ DNA template, and $22 \mu \mathrm{L}$ RNase-free $\mathrm{H}_{2} \mathrm{O}$. OXA-51 and 16S rRNA were used as internal controls. The cycling conditions were as follows: an initial denaturation step of 8 min at $94^{\circ} \mathrm{C}$, followed by 30 cycles of denaturation at $94^{\circ} \mathrm{C}$ for $30 \mathrm{~s}$, annealing at $60^{\circ} \mathrm{C}$ for $30 \mathrm{~s}$, and extension at $72^{\circ} \mathrm{C}$ for $1 \mathrm{~min}$. The purity and integrity of each PCR product was evaluated after separation on $3 \%$ agarose gel electrophoresis and analysis under ultraviolet light.

\section{Antibiotic susceptibility testing}

The antibiotic susceptibility of the $A$. baumannii strains was assessed using a Vitek 2 Compact system (bioMérieux, Inc., Marcy-l'Etoile, France). Three strains were used as control strains, including Escherichia coli (ATCC 25922), Pseudomonas aeruginosa (ATCC 27853), and Staphylococcus aureus (ATCC 29213).

\section{Statistical analysis}

Frequencies were used to describe the distribution of categorical variables and median and interquartile ranges were used for continuous variables. All tests were two-sided and $\mathrm{P}<$ 0.05 was considered significant. Statistical analysis was conducted using the SPSS 16.0 software package (SPSS Inc., Chicago, IL, USA).

\section{RESULTS}

\section{Strain identification}

Of the 205 A. baumannii strains that we collected in total, $130(63.41 \%)$ were isolated from the intensive care unit, 60 (29.27\%) from surgical wards, and $15(7.32 \%)$ from general wards. The samples were obtained from various infection sites: $141(68.78 \%)$ strains were isolated from sputum, $23(11.22 \%)$ from the nose, $22(10.74 \%)$ from blood, $14(6.83 \%)$ from wounds, and the remaining $6(2.44 \%)$ were isolated from other sites.

\section{Detection of carbapenem-resistance genes}

By multiplex PCR, we found that $68(33.17 \%)$ strains were positive for the blaOXA-23 gene. Of these trains, $88.24 \%$ showed resistance to carbapenems while $11.76 \%$ were sensitive to carbapenems (Figure 1). The blaOXA-51 gene was identified in $132(64.39 \%)$ strains, and $17.42 \%$ of these were resistant to carbapenems, while $82.58 \%$ showed sensitivity to carbapenems. In addition, $5(2.44 \%)$ strains were positive for blaOXA-58. Only $80 \%$ of these strains showed resistance to carbapenems and $20 \%$ were sensitivity to carbapenems (Table 2). Moreover, the blaOXA-24 was not detected by multiplex PCR analysis.

\section{Antibiotic susceptibility testing}

We tested the antibiotic susceptibility of the 205 isolated $A$. baumannii strains. We found that $A$. baumannii was $100 \%$ resistant to ampicillin, cefotetan, cefazolin, and cefoperazone (Table 3 ). Moreover, we found that $A$. baumannii exhibited a high resistance $(>50 \%)$ to cefepime, 
piperacillin-tazobactam, amikacin, levofloxacin, ciprofloxacin, tetracycline, ceftazidime, cefotaxime, sulfamethoxazole-trimethoprim, gentamicin, and piperacillin. The drug resistance to cefoperazonesulbactam, meropenem, and imipenem was lower.

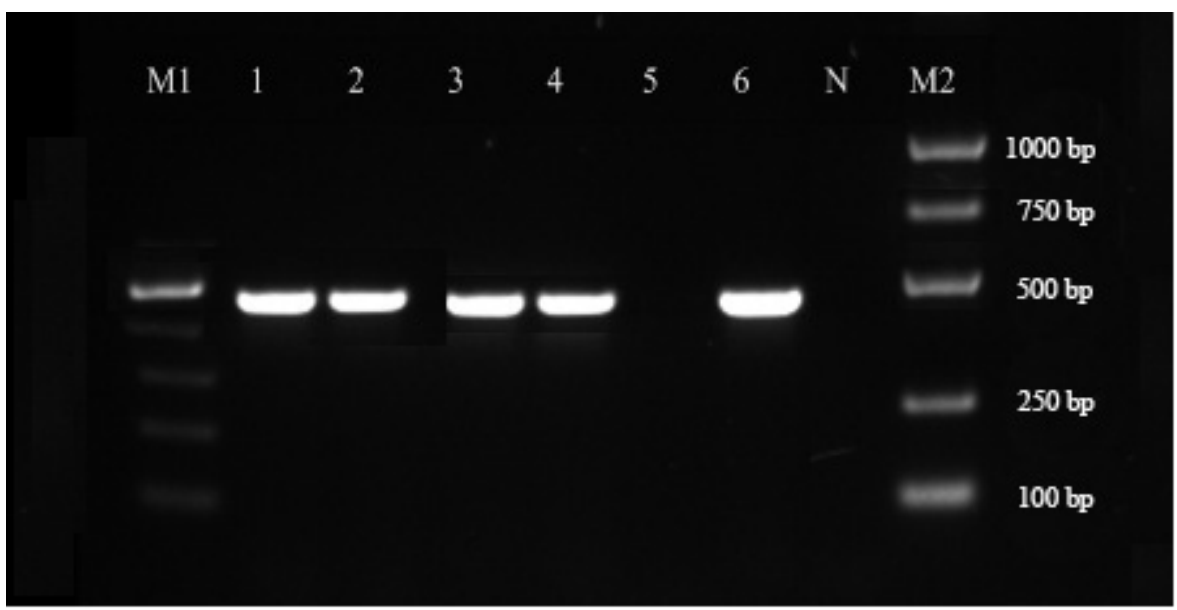

Figure 1. Positive blaOXA-23 gene of PCR test. Lanes 1-4 and $6=$ blaOXA-23; lanes $M 1$ and $M 2=$ molecular markers; lane $N=$ negative control.

Table 2. PCR results for carbapenem-resistance genes.

\begin{tabular}{l|c|c|c|c|c}
\hline OXA genes & Positive PCR & \multicolumn{2}{|c|}{ Carbapenem resistant } & \multicolumn{2}{c}{ Carbapenem sensitive } \\
\hline & $\mathrm{N}$ & $\mathrm{N}$ & $\%$ & $\mathrm{~N}$ & $\%$ \\
\hline blaOXA-23 & 68 & 60 & 88.24 & 8 & 11.76 \\
\hline blaOXA-24 & 0 & 0 & 0.00 & 0 & 0.00 \\
\hline blaOXA-51 & 132 & 23 & 17.42 & 109 & 82.58 \\
\hline blaOXA-58 & 5 & 4 & 80.00 & 1 & 20.00 \\
\hline
\end{tabular}

Table 3. Acinetobacter baumannii drug resistance.

\begin{tabular}{l|c|r}
\hline Antibiotic drug & $\mathrm{N}$ & $\%$ \\
\hline Ampicillin & 205 & 100.00 \\
\hline Cefoperazone-sulbactam & 13 & 100.34 \\
\hline Cefotetan & 205 & 100.00 \\
\hline Cefazolin & 205 & 22.44 \\
\hline Meropenem & 46 & 32.20 \\
\hline Imipenem & 66 & 69.27 \\
\hline Cefepime & 142 & 81.46 \\
\hline Piperacillin-tazobactam & 167 & 80.49 \\
\hline Amikacin & 165 & 75.12 \\
\hline Levofloxacin & 154 & 83.90 \\
\hline Ciprofloxacin & 172 & 84.88 \\
\hline Tetracycline & 174 & 86.83 \\
\hline Ceftazidime & 178 & 87.32 \\
\hline Cefotaxime & 179 & 100.00 \\
\hline Cefoperazone & 205 & 84.39 \\
\hline Sulfamethoxazole-trimethoprim & 173 & 92.68 \\
\hline Gentamicin & 190 & 96.59
\end{tabular}




\section{DISCUSSION}

The non-fermenting, aerobic bacillus $A$. baumannii is an opportunistic pathogen that causes nosocomial infections, especially in intensive care units. $A$. baumannii easily spreads from patient to patient in hospitals, for example via medical equipment, and the infection is hard to control because of high drug resistance (Bergogne-Bérézin and Towner, 1996; Abbo et al., 2005). Carbapenems are generally used to treat patients suffering from $A$. baumannii infections, as they are currently the most active antibiotics. However, through the production of carbapenemase and the acquisition of carbapenem-hydrolyzing $\beta$-lactamases of the Ambler class B and D (Poirel and Nordmann, 2006; Queenan and Bush, 2007), A. baumannii can acquire resistance to carbapenems as well. The blaOXA-23, blaOXA-24, blaOXA-51, and blaOXA-58 genes encode four Ambler class $\mathrm{D} \beta$-lactamases, and the present study investigated their role in the drug resistance of $A$. baumannii.

We found that the blaOXA-23- and blaOXA-51-resistance genes were most frequently present in $A$. baumannii isolates, and that blaOXA-23 is mainly responsible for resistance to carbapenems. Previous studies have reported similar results (Jeannot et al., 2014; Santimaleeworagun et al., 2014; Khorsi et al., 2015; Mathlouthi et al., 2015; Memish et al., 2015; Wang et al., 2015). Khorsi et al. (2015) analyzed the prevalence of $A$. baumannii multidrugresistance in hospitals in Algiers, and found that carbapenem resistance was mainly mediated by blaOXA-23 and blaOXA-24 genes. Wang et al. (2015) also identified the blaOXA-23 gene in multidrug-resistant $A$. baumannii isolates from Libyan hospitals. Memish et al. (2015) have reported that blaOXA-23 is the dominant carbapenemase in A. baumannii. Jeannot et al. (2014) reported that blaOXA-23 was the most frequently acquired gene in A. baumannii. In contrast with these findings, Ma et al. (2015) reported that blaOXA51 and blaOXA58 genes were most frequently detected in $A$. baumannii. These authors did not identify blaOXA-23 in samples collected at a Chinese hospital. The discrepancies between the findings in these studies may be explained by differences in sample selection or by genetic variation among the different populations.

Our study shows that $100 \%$ of the $A$. baumannii isolated strains is resistant to ampicillin, cefotetan, cefazolin, and cefoperazone. In addition, $A$. baumannii is highly resistant to cefepime, piperacillin-tazobactam, amikacin, levofloxacin, ciprofloxacin, tetracycline, ceftazidime, cefotaxime, sulfamethoxazole-trimethoprim, gentamicin, and piperacillin. Previous studies have reported similar results (Vakili et al., 2014; Ma et al., 2015; Zhao et al., 2015). Zhao et al. (2015) reported that the most important drug resistance genes of $A$. baumannii are blaOXA-51 and blaOXA-23, conferring multidrug resistance to the bacterial strain.

In summary, our study suggests that the blaOXA-23 and blaOXA-51 genes are most frequently present in $A$. baumannii, and that resistance to carbapenems is mainly correlated with blaOXA-23 gene expression. Further molecular analysis of $A$. baumannii could help to identify common sources of infection, and to prevent its spread in hospitals.

\section{Conflicts of interest}

The authors declare no conflict of interest.

\section{ACKNOWLEDGMENTS}

We acknowledged the great help from staffs in Nanyang City Center Hospital for collecting the blood samples for analysis. 


\section{REFERENCES}

Abbo A, Navon-Venezia S, Hammer-Muntz O, Krichali T, et al. (2005). Multidrug-resistant Acinetobacter baumannii. Emerg. Infect. Dis. 11: 22-29. http://dx.doi.org/10.3201/eid1101.040001

Bergogne-Bérézin E and Towner KJ (1996). Acinetobacter spp. as nosocomial pathogens: microbiological, clinical, and epidemiological features. Clin. Microbiol. Rev. 9: 148-165.

Boucher HW, Talbot GH, Bradley JS, Edwards JE, et al. (2009). Bad bugs, no drugs: no ESKAPE! An update from the Infectious Diseases Society of America. Clin. Infect. Dis. 48: 1-12. http://dx.doi.org/10.1086/595011

Dijkshoorn L, Nemec A and Seifert H (2007). An increasing threat in hospitals: multidrug-resistant Acinetobacter baumannii. Nat. Rev. Microbiol. 5: 939-951. http://dx.doi.org/10.1038/nrmicro1789

Gaynes R and Edwards JR; National Nosocomial Infections Surveillance System (2005). Overview of nosocomial infections caused by Gram-negative bacilli. Clin. Infect. Dis. 41: 848-854. http://dx.doi.org/10.1086/432803

Jeannot K, Diancourt L, Vaux S, Thouverez M, et al. (2014). Molecular epidemiology of carbapenem non-susceptible Acinetobacter baumannii in France. PLoS One 9: e115452. http://dx.doi.org/10.1371/journal.pone.0115452

Jeong SH, Bae IK, Park KO, An YJ, et al. (2006). Outbreaks of imipenem-resistant Acinetobacter baumannii producing carbapenemases in Korea. J. Microbiol. 44: 423-431.

Khorsi K, Messai Y, Hamidi M, Ammari H, et al. (2015). High prevalence of multidrug-resistance in Acinetobacter baumannii and dissemination of carbapenemase-encoding genes blaOXA-23-like, blaOXA-24-like and blaNDM-1 in Algiers hospitals. Asian Pac. J. Trop. Med. 8: 438-446. http://dx.doi.org/10.1016/j.apjtm.2015.05.011

Ma Z, Zhou L, Wang H and Luo L (2015). Investigation on the genomic diversity of OXA from isolated Acinetobacter baumannii. Int. J. Clin. Exp. Med. 8: 4429-4432.

Mathlouthi N, Areig Z, Al Bayssari C, Bakour S, et al. (2015). Emergence of Carbapenem-Resistant Pseudomonas aeruginosa and Acinetobacter baumannii Clinical Isolates Collected from Some Libyan Hospitals. Microb. Drug Resist. 21: 335-341. http://dx.doi.org/10.1089/mdr.2014.0235

Memish ZA, Assiri A, Almasri M, Roshdy H, et al. (2015). Molecular characterization of carbapenemase production among Gram-negative bacteria in Saudi Arabia. Microb. Drug Resist. 21: 307-314. http://dx.doi.org/10.1089/mdr.2014.0121

Peleg AY, Seifert H and Paterson DL (2008). Acinetobacter baumannii: emergence of a successful pathogen. Clin. Microbiol. Rev. 21: 538-582. http://dx.doi.org/10.1128/CMR.00058-07

Peymani A, Higgins PG, Nahaei MR, Farajnia S, et al. (2012). Characterisation and clonal dissemination of OXA-23-producing Acinetobacter baumannii in Tabriz, northwest Iran. Int. J. Antimicrob. Agents 39: 526-528. http://dx.doi.org/10.1016/j. ijantimicag.2012.02.014

Poirel L and Nordmann P (2006). Carbapenem resistance in Acinetobacter baumannii: mechanisms and epidemiology. Clin. Microbiol. Infect. 12: 826-836. http://dx.doi.org/10.1111/j.1469-0691.2006.01456.x

Queenan AM and Bush K (2007). Carbapenemases: the versatile beta-lactamases. Clin. Microbiol. Rev. 20: 440-458. http:// dx.doi.org/10.1128/CMR.00001-07

Santimaleeworagun W, Thathong A, Samret W, Preechachuawong P, et al. (2014). Identification and characterization of carbapenemase genes in clinical isolates of carbapenem-resistant Acinetobacter baumannii from general hospital in Thailand. Southeast Asian J. Trop. Med. Public Health 45: 874-880.

Vakili B, Fazeli H, Shoaei P, Yaran M, et al. (2014). Detection of colistin sensitivity in clinical isolates of Acinetobacter baumannii in Iran. J. Res. Med. Sci. 19 (Suppl 1): S67-S70.

Wang D, Ma L, Wu Z, Li M, et al. (2015). Identification and characteristics of imipenem-resistant Acinetobacter baumannii in surgical wards in a Chinese university hospital. Infect Dis (Lond) 47: 182-186. http://dx.doi.org/10.3109/00365548.2014 $\underline{.979435}$

Yang HY, Lee HJ, Suh JT and Lee KM (2009). Outbreaks of imipenem resistant Acinetobacter baumannii producing OXA-23 beta-lactamase in a tertiary care hospital in Korea. Yonsei Med. J. 50: 764-770. http://dx.doi.org/10.3349/ ymi.2009.50.6.764

Zhao SY, Jiang DY, Xu PC, Zhang YK, et al. (2015). An investigation of drug-resistant Acinetobacter baumannii infections in a comprehensive hospital of East China. Ann. Clin. Microbiol. Antimicrob. 14: 7. http://dx.doi.org/10.1186/s12941-015$\underline{0066-4}$ 